PROCEEDINGS OF THE

AMERICAN MATHEMATICAL SOCIETY

Volume 130, Number 3, Pages 883-892

S 0002-9939(01)06095-6

Article electronically published on August 28, 2001

\title{
ON THE MAXIMAL INEQUALITIES FOR MARTINGALES INVOLVING TWO FUNCTIONS
}

\author{
MEI TAO AND PEIDE LIU
}

(Communicated by Claudia M. Neuhauser)

\begin{abstract}
Let $\Phi(t)$ and $\Psi(t)$ be nonnegative convex functions, and let $\varphi$ and $\psi$ be the right continuous derivatives of $\Phi$ and $\Psi$, respectively. In this paper, we prove the equivalence of the following three conditions: (i) $\left\|f^{*}\right\|_{\Phi} \leq$ $c\|f\|_{\Psi}$, (ii) $L^{\Psi} \subseteq H^{\Phi}$ and (iii) $\int_{s_{0}}^{t} \frac{\varphi(s)}{s} d s \leq c \psi(c t), \forall t>s_{0}$, where $L^{\Psi}$ and $H^{\Phi}$ are the Orlicz martingale spaces. As a corollary, we get a sufficient and necessary condition under which the extension of Doob's inequality holds. We also discuss the converse inequalities.
\end{abstract}

\section{INTRODUCTION}

Let $\Phi$ be a nonnegative convex continuous function on $[0, \infty)$ with $\Phi(0)=0$ and $\lim _{t \rightarrow \infty} \Phi(t)=\infty$, and $(\Omega, \mathcal{F}, \mu)$ be a complete probability space. We denote by $\mathcal{M}$ the set of all $\mathcal{F}$-measurable functions and put

$$
L^{\Phi}(\Omega)=\{f \in \mathcal{M}, \exists \epsilon>0, E \Phi(\epsilon|f|)<\infty\}
$$

where $E$ stands for the expectation with respect to $\mu . L^{\Phi}(\Omega)$ is the so-called Orlicz space (see Rao [1] $)$. Define $\|f\|_{\Phi}=\inf \left\{k>0, E \Phi\left(\frac{|f|}{k}\right) \leq 1\right\}$. Let $\mathcal{F}_{n}(n \geq 1)$ be a nondecreasing sequence of complete sub- $\sigma$-fields and $\mathcal{F}=\bigvee_{n=0}^{\infty} \mathcal{F}_{n}$. Denote the maximal function and the $\Phi$-norm of a submartingale $f=\left(f_{n}\right)_{n \geq 0}$ adapted to $\mathcal{F}_{n}$ $(n \geq 1)$ respectively by

$$
f^{*}(\omega)=\sup _{n \geq 0}\left|f_{n}(\omega)\right|, \quad\|f\|_{\Phi}=\sup _{n \geq 0}\left\|f_{n}\right\|_{\Phi} .
$$

It is well known that if $\Phi$ is a strictly convex function on $[0, \infty)$, i.e. $q_{\Phi}=\inf _{t>0} \frac{t \varphi(t)}{\Phi(t)}$ $>1$ (where $\varphi$ is the right continuous derivative of $\Phi$ ), the extension of the classical Doob's inequality

$$
\left\|f^{*}\right\|_{\Phi} \leq c \sup _{n \geq 0}\left\|f_{n}\right\|_{\Phi}
$$

holds for every martingale or nonnegative submartingale $f=\left(f_{n}\right)_{n \geq 0}$; here $c$ is a constant depending only on $\Phi$. When $\Phi$ is not strictly convex, the situation

Received by the editors February 4, 2000 and, in revised form, August 25, 2000.

2000 Mathematics Subject Classification. Primary 60G42, 43A17.

Key words and phrases. Martingale inequality, nonnegative submartingale, maximal function, Orlicz space.

This research was supported by the National Science Foundation of the People's Republic of China.

(C)2001 American Mathematical Society 
is rather different. To see this, recall Doob's inequality in the case of $p=1$ and Imkeller's inequality [4]

$$
E f^{*} \leq \frac{e}{e-1}\left(1+\sup _{n \geq 0} E\left|f_{n}\right| \log ^{+}\left|f_{n}\right|\right), \quad\left\|f^{*}\right\|_{1} \leq c \sup _{n \geq 0}\left\|f_{n}\right\|_{\Psi},
$$

where $\Psi=t \log ^{+} t$, which hold for every martingale or nonnegative submartingale $f$. It means that $f^{*} \in L^{1}$ if $f \in L \log ^{+} L$.

In [5]-[7], Kita considered some conditions about two such functions $\Phi$ and $\Psi$, for which the inequality

$$
\int_{R^{n}} \Phi\left(f^{*}(x)\right) d x \leq c \int_{R^{n}} \Psi(c|f(x)|) d x
$$

holds for every function $f \in L^{\Psi}$ defined in $R^{n}$, where $f^{*}$ is the Hardy-Littlewood maximal function of $f$. In particular, he observed that condition (1) holds for every function $f \in L^{\Psi}$ if and only if $\int_{0}^{t} \frac{\varphi(s)}{s} d s \leq c \psi(c t), \forall t>0$, where $\varphi, \psi$ stand for the right continuous derivatives of $\Phi$ and $\Psi$, respectively and $c>0$ is some constant. He also considered the condition for which the converse inequality holds. Moreover, the authors noticed that D. Gilat 2 had obtained a sharp inequality that gives a comparison of the $L_{p}$ norm of a martingale to the $L_{1}$ norm of its maximal function.

In this paper, we consider the Orlicz spaces of martingales and prove the equivalence of the following three conditions : (i) $\left\|f^{*}\right\|_{\Phi} \leq c\|f\|_{\Psi}$ for every martingale or nonnegative submartingale $f$, (ii) $L^{\Psi} \subseteq H^{\Phi}$, (iii) $\int_{s_{0}}^{t} \frac{\varphi(s)}{s} d s \leq c \psi(c t), \forall t>s_{0}$, where $s_{0}, c$ are some positive constants and the Orlicz martingale spaces $L^{\Phi}$ and $H^{\Phi}$ are defined as

$$
\begin{gathered}
L^{\Phi}=\left\{f=\left(f_{n}\right)_{n \geq 0}:\|f\|_{\Phi}<\infty\right\}, \\
H^{\Phi}=\left\{f=\left(f_{n}\right)_{n \geq 0}:\|f\|_{H^{\Phi}}=\left\|f^{*}\right\|_{\Phi}<\infty\right\} .
\end{gathered}
$$

As a corollary, a sufficient and necessary condition under which the extension of Doob's inequality holds is obtained. We also consider the converse inequalities for the regular martingales.

In this paper $|A|$ always means the measure of set $A$ with respect to $\mu$.

\section{The maximal inequalities}

Lemma 1. Let $f=\left(f_{n}\right)_{n \geq 0}$ be a nonnegative submartingale. Then

$$
\left|\left\{f_{n}^{*}>t\right\}\right| \leq \frac{2}{t} \int_{\frac{t}{2}}^{\infty}\left|\left\{f_{n}>\lambda\right\}\right| d \lambda, \quad \forall t>0, n \in N,
$$

and

$$
\int_{\frac{t}{2}}^{\infty}\left|\left\{f_{n}>\lambda\right\}\right| d \lambda \leq \int_{\left\{f_{n}>\frac{t}{2}\right\}} f_{n} d \mu .
$$

Proof. For $t>0$, let $h_{n}=\left(f_{n} \vee \frac{t}{2}\right)-\frac{t}{2}$ for every $n \in N$. It is easy to see that $h=\left(h_{n}\right)_{n \geq 0}$ is a nonnegative submartingale. By using Kolmogorof's inequality and Fubini's theorem we have

$$
\begin{aligned}
\left|\left\{f_{n}^{*}>t\right\}\right| & =\left|\left\{h_{n}^{*}>\frac{t}{2}\right\}\right| \leq \frac{2}{t} \int_{\Omega} h_{n} d \mu=\frac{2}{t} \int_{\Omega}\left(f_{n} \vee \frac{t}{2}\right) d \mu-1 \\
& =\frac{2}{t} \int_{0}^{\infty}\left|\left\{f_{n} \vee \frac{t}{2}>\lambda\right\}\right| d \lambda-1=\frac{2}{t} \int_{\frac{t}{2}}^{\infty}\left|\left\{f_{n}>\lambda\right\}\right| d \lambda
\end{aligned}
$$


which implies (2). From this equation we get

$$
\frac{2}{t} \int_{\frac{t}{2}}^{\infty}\left|\left\{f_{n}>\lambda\right\}\right| d \lambda=\frac{2}{t} \int_{\Omega}\left(f_{n} \vee \frac{t}{2}\right) d \mu-1 \leq \frac{2}{t} \int_{\left\{f_{n}>\frac{t}{2}\right\}} f_{n} d \mu
$$

which implies (3).

Theorem 1. Suppose that $\Phi, \Psi$ are nondecreasing convex functions defined on $[0, \infty)$ with $\Phi(0)=\Psi(0)=0$. Then the following statements are equivalent:

(i) There exist $s_{0}, c_{1}>0$ such that

$$
\int_{s_{0}}^{t} \frac{\varphi(s)}{s} d s \leq c_{1} \psi\left(c_{1} t\right), \quad \forall t>s_{0} .
$$

(ii) There exists $c_{4}>0$ such that

$$
\left\|f^{*}\right\|_{\Phi} \leq c_{4}\|f\|_{\Psi}
$$

for every martingale or nonnegative submartingale $f=\left(f_{n}\right)_{n \geq 0}$.

(iii) $L^{\Psi} \subseteq H^{\Phi}$

Proof. (i) $\Longrightarrow$ (ii). We only prove (5) for nonnegative submartingales. By using Lemma 1 and Fubini's theorem we get

$$
\begin{aligned}
E \Phi\left(f_{n}^{*}\right) & =\int_{0}^{\infty}\left|\left\{f_{n}^{*}>t\right\}\right| d \Phi(t) \\
& \leq \int_{s_{0}}^{\infty}\left|\left\{2 f_{n}^{*}>2 t\right\}\right| d \Phi(t)+\Phi\left(s_{0}\right) \\
& \leq \int_{s_{0}}^{\infty} \frac{1}{t} \int_{t}^{\infty}\left|\left\{2 f_{n}>\lambda\right\}\right| d \lambda d \Phi(t)+\Phi\left(s_{0}\right) \\
& =\int_{s_{0}}^{\infty}\left|\left\{2 f_{n}>\lambda\right\}\right| \int_{s_{0}}^{\lambda} \frac{1}{t} d \Phi(t) d \lambda+\Phi\left(s_{0}\right) \\
& \leq E \Psi\left(2 c_{1} f_{n}\right)+\Phi\left(s_{0}\right) .
\end{aligned}
$$

Without loss of generality, we assume that $\left\|2 c_{1} f\right\|_{\Psi}=1$. Then $E \Phi\left(f^{*}\right) \leq 1+\Phi\left(s_{0}\right)$ and $E \Phi\left(\frac{f^{*}}{1+\Phi\left(s_{0}\right)}\right) \leq 1$. Hence

$$
\left\|f^{*}\right\|_{\Phi} \leq 1+\Phi\left(s_{0}\right) \leq c_{4}\|f\|_{\Psi},
$$

where $c_{4}=2\left(1+\Phi\left(s_{0}\right)\right) c_{1}$, as desired.

(ii) $\Longrightarrow$ (iii) is obvious.

$($ iii $) \Longrightarrow($ i). First we prove that $(5)$ holds for every nonnegative martingale $f=\left(f_{n}\right)_{n \geq 0}$ with $\|f\|_{\Psi}=1$. Assume it is not the case. Then for every $n$, choose a nonnegative martingale $f^{(n)}=\left(f_{n k}\right)_{k \geq 0}$ defined on $\left(\Omega_{n}, \mathcal{F}_{n}, \mathcal{F}_{n k}, \mu_{n}\right)$ satisfying $\left\|f^{(n)}\right\|_{\Psi}=1,\left\|f^{(n) *}\right\|_{\Phi}>4^{n}$. Consider the product space $\left(\Omega, \mathcal{F}, \mathcal{F}_{k}, \mu\right)=$ $\prod_{n=1}^{\infty}\left(\Omega_{n}, \mathcal{F}_{n}, \mathcal{F}_{n k}, \mu_{n}\right)$, where $\mathcal{F}_{k}=\prod_{n=1}^{\infty} \mathcal{F}_{n k}$ and take $h^{(n)}=\left(h_{n k}\right)_{k \geq 1}$, where $h_{n k}=\chi_{\Omega_{1}} \times \cdots \times \chi_{\Omega_{n-1}} \times f_{n k} \times \chi_{\Omega_{n+1}} \times \cdots$. It is easy to see that $h^{(n)}$ is a martingale with $\left\|h^{(n)}\right\|_{\Psi}=1$ and $\left\|h^{(n)^{*}}\right\|_{\Phi}>4^{n}$. Let $g_{k}(\omega, t)=\sum_{n=1}^{\infty} \frac{h_{n k}}{2^{n}}$. Then $\|g\|_{\Psi} \leq \sum_{n=1}^{\infty}\left\|\frac{h_{n k}}{2^{n}}\right\|_{\Psi}=1$ and $\left\|\frac{g^{*}}{2^{n}}\right\|_{\Phi} \geq\left\|\frac{h^{(n)^{*}}}{4^{n}}\right\|_{\Phi}>1, \forall n>0$. This contradicts the fact that $g \in L^{\Psi} \subseteq H^{\Phi}$.

To prove (i), let $A_{k}=\left(0, \frac{1}{2^{k}}\right], \mathcal{F}_{k}=\sigma\left\{A_{0}, A_{1}, A_{2}, \cdots, A_{k}\right\}, f=t \chi_{A_{n}}, f=$ $\left(E\left(f \mid \mathcal{F}_{k}\right)\right)_{k \geq 0}$, where $k, n \geq 0, t=\Psi^{-1}\left(2^{n}\right)$. Then $f$ is a finite dyadic martingale 
on $(0,1]$ with $\frac{t}{2^{n}} \leq f^{*}(\omega) \leq t$. It is clear that $\|f\|_{\Psi}=1$ and then $\left\|f^{*}\right\|_{\Phi} \leq$ $c_{4}\|f\|_{\Psi}=c_{4}$. Thus $E \Phi\left(\frac{f^{*}}{c_{4}}\right) \leq 1$, i.e.

$$
\int_{0}^{\infty}\left|\left\{f^{*}>c_{4} s\right\}\right| \varphi(s) d s \leq 1 .
$$

Notice that $\frac{1}{s} \leq \frac{c_{4} 2^{n-k}}{t}$ and $\left|\left\{f^{*}>c_{4} s\right\}\right|=\frac{1}{2^{k+1}}$ when $s \in\left(\frac{t}{c_{4} 2^{n-k}}, \frac{t}{c_{4} 2^{n-k-1}}\right] \quad(0 \leq$ $k \leq n-1)$. We have

$$
\frac{1}{s} \leq \frac{c_{4} 2^{n+1}}{t} \cdot \frac{1}{2^{k+1}}=\frac{c_{4} 2^{n+1}}{t} \cdot\left|\left\{f^{*}>c_{4} s\right\}\right|, \quad \forall s \in\left(\frac{t}{c_{4} 2^{n}}, \frac{t}{c_{4}}\right] .
$$

From (7) we get

$$
\int_{\frac{t}{c_{4} 2^{n}}}^{\frac{t}{c_{4}}} \frac{\varphi(s)}{s} d s \leq \frac{c_{4} 2^{n+1}}{t} \int_{0}^{\infty}\left|\left\{f^{*}>c_{4} s\right\}\right| \varphi(s) d s \leq \frac{c_{4} 2^{n+1}}{t} \leq \frac{2 c_{4} \Psi(t)}{t} \leq 2 c_{4} \psi(t) .
$$

By the convexity of $\Psi$, we have $\frac{t}{c_{4} 2^{n}} \leq \frac{\Psi^{-1}(1)}{c_{4}}=s_{0}, \forall n \geq 0$, and then $\int_{s_{0}}^{\frac{t}{c_{4}}} \frac{\varphi(s)}{s} d s \leq$ $2 c_{4} \psi(t)$,

$$
\begin{aligned}
\int_{s_{0}}^{u} \frac{\varphi(s)}{s} d s & \leq 2 c_{4} \psi\left(\Psi^{-1}\left(2^{n+1}\right)\right) \\
& \leq 2 c_{4} \psi\left(2 c_{4} u\right), \quad \forall u \in\left(\frac{1}{c_{4}} \Psi^{-1}\left(2^{n}\right), \frac{1}{c_{4}} \Psi^{-1}\left(2^{n+1}\right)\right], n \geq 0 .
\end{aligned}
$$

That is,

$$
\int_{s_{0}}^{t} \frac{\varphi(s)}{s} d s \leq c_{1} \psi\left(c_{1} t\right), \quad \forall t \geq s_{0}
$$

where $c_{1}=2 c_{4}$. This completes the proof.

Letting $\Phi=\Psi$ in Theorem 1, we get

Corollary 1. Suppose that $\Phi$ is a nondecreasing convex function defined on $[0, \infty)$ with $\Phi(0)=0$. Then

$$
\left\|f^{*}\right\|_{\Phi} \leq \sup _{n}\left\|f_{n}\right\|_{\Phi}
$$

holds for every martingale or nonnegative submartingale $f=\left(f_{n}\right)_{n \geq 0}$ if and only if there exist $s_{0}, c_{1}>0$ such that

$$
\int_{s_{0}}^{t} \frac{\varphi(s)}{s} d s \leq c_{1} \varphi\left(c_{1} t\right), \forall t>s_{0} .
$$

We will prove in Section 3 that (8) is equivalent to the condition $\underline{\lim }_{t \rightarrow \infty} \frac{t \varphi(t)}{\Phi(t)}>1$ when $\Phi \in \Delta_{2}$ (i.e. there exist $c, t_{0}>0$ such that $\Phi(2 t) \leq c \Phi(t), \forall t>t_{0}$ ).

Stein [12] proved that if $f$ is a function supported in a ball $B \subseteq R^{n}$, then $f^{*} \in L^{1}(B)$ implies $f \in L \log ^{+} L$. Kita [7] proved some similar results on the Orlicz function spaces. The following theorem shows that the situation for martingales is different, i.e. for martingale space, it can happen that $H_{+}^{1} \not \subset L \log ^{+} L$.

Theorem 2. Suppose that $\Phi, \Psi$ are nondecreasing convex functions defined on $[0, \infty)$ with $\Phi(0)=\Psi(0)=0$. Then the following statements are equivalent:

(i) There exists $c>0$ such that $\varliminf_{t \rightarrow \infty} \frac{\Phi(c t)}{\Psi(t)}>0$.

(ii) $H^{\Phi} \subseteq L^{\Psi}$. 
(iii) $H_{+}^{\Phi} \subseteq L_{+}^{\Psi}$, where $H_{+}^{\Phi}$ and $L_{+}^{\Psi}$ are the sets of all positive members of $H^{\Phi}$, $L^{\Psi}$, respectively.

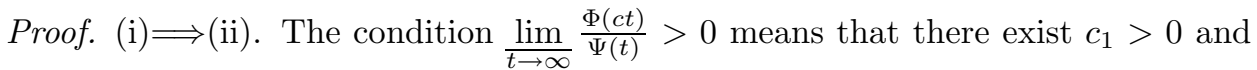
$n_{0} \in N$ such that $\Phi\left(c_{1} t\right)>\Psi(t), \forall t>n_{0}$. For $f \in H^{\Phi}$, there exists $k>0$ such that $E \Phi\left(\frac{f^{*}}{k}\right)<M<\infty$, and then

$$
E \Psi\left(\frac{f}{c_{1} k}\right)<\Psi\left(t_{0}\right)+E_{\left\{\frac{f}{c_{1} k}>n_{0}\right\}} \Phi\left(\frac{f}{k}\right)<\Psi\left(t_{0}\right)+M<\infty ;
$$

hence $f \in L^{\Psi}$. It shows that $H^{\Phi} \subseteq L^{\Psi}$.

(ii) $\Longrightarrow$ (iii) is obvious.

(iii) $\Longrightarrow$ (i). When $H_{+}^{\Phi} \subseteq L_{+}^{\Psi}$, it follows from the proof of (iii) $\Longrightarrow$ (i) in Theorem 1 that there exists $c>0$ such that

$$
\|f\|_{\Psi} \leq c\left\|f^{*}\right\|_{\Phi}
$$

for every nonnegative martingale $f=\left(f_{n}\right)_{n \geq 0} \in H^{\Phi}$ defined on $\left(\Omega, \mathcal{F}, \mathcal{F}_{n}, \mu\right)$. Now, for every such a martingale $f$ with $E \Psi(f) \geq 1$, define $g=\left(g_{m}\right)_{m>0}$ on the product space $\left(\Omega \times(0,1], \mathcal{F}_{n} \times \sigma\{(0, \alpha]\}, \mu \times \nu\right)$ :

$$
g_{m}(\omega, t):=f_{m}(\omega) \chi_{(0, \alpha]}(t), \quad \forall t \in(0,1],
$$

where $\nu$ is Lebesgue measure on $(0,1]$ and $\alpha=\frac{1}{E \Psi(f)}$. Denote by $\tilde{E}$ the expectation with respect to $\Omega \times(0,1]$. Then $\tilde{E} \Psi(g)=\alpha E \Psi(f)=1$ and $\left\|c g^{*}\right\|_{\Phi} \geq\|g\|_{\Psi}=1$. It follows that $\tilde{E} \Phi\left(c g^{*}\right) \geq 1$ and then

$$
E \Phi\left(c f^{*}\right) \geq E \Psi(f) .
$$

Let $t \geq \Psi^{-1}(1)$ and $f \equiv t$. Then $E \Psi(f) \geq 1$. From (9) we have $\Phi(c t) \geq \Psi(t), \forall t \geq$ $\Psi^{-1}(1)$. This implies that $\underline{\lim }_{t \rightarrow \infty} \frac{\Phi(c t)}{\Psi(t)}>0$.

Corollary 2. Suppose that $\Phi, \Psi$ are nondecreasing convex functions on $[0, \infty)$ with $\Phi(0)=\Psi(0)=0$. Then $H^{\Phi}=L^{\Psi}$ if and only if $H^{\Psi}=L^{\Phi}$.

Proof. We only prove the sufficiency, and the necessity can be obtained from the "symmetry". Indeed, from $H^{\Phi}=L^{\Psi}$ and Theorems 1 and 2 , there exist $s_{0}, c_{1}>0$ such that

$$
\int_{s_{0}}^{t} \frac{\varphi(s)}{s} d s \leq c_{1} \psi\left(c_{1} t\right), \quad \Phi\left(c_{1} t\right)>\Psi(t), \quad \forall t>s_{0} .
$$

A simple computation shows that $c_{1} \varphi\left(c_{1} t\right)>\frac{t}{2} \psi\left(\frac{t}{2}\right), \forall t>s_{0}$, and

$$
\int_{c_{1} s_{0}}^{t} \frac{\psi\left(\frac{s}{2 c_{1}}\right)}{s} d s \leq \int_{c_{1} s_{0}}^{t} \frac{\varphi(s)}{s} d s \leq c_{1} \psi\left(c_{1} t\right) \leq 2 c_{1}^{2} \varphi\left(2 c_{1}^{2} t\right), \quad \forall t>c_{1} s_{0} ;
$$

therefore

$$
\int_{s_{1}}^{t} \frac{\psi(s)}{s} d s \leq c \varphi(c t), \quad \forall t>s_{1}
$$

where $s_{1}=\frac{s_{0}}{2}, c=2 c_{1}^{2}$. Thus $H^{\Psi} \supseteq L^{\Phi}$. The converse relation comes from $H^{\Phi}=L^{\Psi}$ directly, and hence $H^{\Psi}=L^{\Phi}$ is proved. 
Now we consider regular martingales. Recall that an increasing sequence $\left(\mathcal{F}_{n}\right)_{n \geq 0}$ of sub- $\sigma$ - fields $\left(\mathcal{F}_{0}=\{\emptyset, \Omega\}\right)$ is said to be regular if there exists a $d_{\left(\mathcal{F}_{n}\right)}>0$ such that

$$
\chi(F) \leq d_{\left(\mathcal{F}_{n}\right)} E\left(\chi(F) \mid \mathcal{F}_{n-1}\right), \quad \forall F \in \mathcal{F}_{n}, \quad n \in N .
$$

We say that a probability space $\left(\Omega, \mathcal{F}, \mathcal{F}_{n}, \mu\right)$ (a submartingale $\left.f=\left(f_{n}, \mathcal{F}_{n}\right)_{n \geq 0}\right)$ is regular if $\left(\mathcal{F}_{n}\right)_{n \geq 0}$ is regular.

Lemma $2\left([\underline{8})\right.$. Let $f=\left(f_{n}\right)_{n \geq 0}$ be a regular nonnegative martingale with the constant $d_{\left(\mathcal{F}_{n}\right)}$. Then

$$
\int_{\left\{f_{n}^{*}>\lambda\right\}} f_{n} d \mu \leq d_{\left(\mathcal{F}_{n}\right)} \lambda\left|\left\{f_{n}^{*}>\lambda\right\}\right|, \quad \forall \lambda \geq\left\|f_{0}\right\|_{\infty} .
$$

Theorem 3. Suppose that $\Phi, \Psi$ are nondecreasing convex functions defined on $[0, \infty)$ with $\Phi(0)=\Psi(0)=0$. Then the following statements are equivalent:

(i) There exist $s_{0}, c_{1}>0$ such that

$$
\int_{1}^{t} \frac{\varphi(s)}{s} d s \geq c_{1} \psi\left(c_{1} t\right), \quad \forall t>s_{0} .
$$

(ii) $f \in H^{\Phi}$ implies that $f \in L^{\Psi}$ for every nonnegative regular martingale $f=\left(f_{n}\right)_{n \geq 0}$.

(iii) There exists $c_{2}>0$ such that

$$
c_{2}\left\|f^{*}\right\|_{\Phi} \geq \sup _{n}\left\|f_{n}\right\|_{\Psi}
$$

for every nonnegative regular martingale $f=\left(f_{n}\right)_{n \geq 0}$, where $c_{2}$ depends only on $d_{\left(\mathcal{F}_{n}\right)}$.

Proof. (i) $\Longrightarrow$ (ii). For a nonnegative regular martingale $f \in H^{\Phi}$, choose $c>s_{0}$ such that $\|f\|_{1} \leq c$. By using Fubini's theorem and Lemmas 1 and 2 we get

$$
\begin{aligned}
E \Psi\left(c_{1}|f|\right) & =\int_{0}^{\infty}|\{|f|>s\}| c_{1} \psi\left(c_{1} s\right) d s \\
& \leq \int_{s_{0}}^{\infty}|\{|f|>s\}|\left(\int_{1}^{s} \frac{\varphi(t)}{t} d t\right) d s+\Psi\left(c_{1} c\right) \\
& \leq \int_{1}^{\infty} \frac{\varphi(t)}{t}\left(\int_{t}^{\infty}|\{|f|>s\}| d s\right) d t+\Psi\left(c_{1} c\right) \\
& \leq \int_{1}^{\infty} \frac{\varphi(t)}{t}\left(\int_{|f|>t}|\{|f|>s\}| d s\right) d t+\Psi\left(c_{1} c\right) \\
& \leq \int_{1}^{\infty} \frac{\varphi(t)}{t} d_{\left(\mathcal{F}_{n}\right)} t\left\{f^{*}>t\right\} d t+\Psi\left(c_{1} c\right) \\
& \leq d_{\left(\mathcal{F}_{n}\right)} E \Phi\left(f^{*}\right)+\Psi\left(c_{1} c\right)<\infty .
\end{aligned}
$$

Hence $f \in L^{\Psi}$.

(ii) $\Longrightarrow$ (iii). Without loss of generality, we assume that $\left\|f^{*}\right\|_{\Phi}=1$. If the assertion of (iii) is not true, we can choose a sequence of nonnegative martingales $f^{(n)}=\left(f_{n m}\right)_{m \geq 0}$ defined on $\left(\Omega, \mathcal{F}, \mathcal{F}_{n}, \mu\right)$ such that $4^{n}\left\|f^{(n) *}\right\|_{\Phi}<\left\|f^{(n)}\right\|_{\Psi}$. Let $g_{k}(\omega, t)=\sum_{n=1}^{\infty} \frac{f_{n k}}{2^{n}}$. Then $\left\|g^{*}\right\|_{\Phi} \leq \sum_{n=1}^{\infty}\left\|\frac{f^{(n)^{*}}}{2^{n}}\right\|_{\Phi}=1$ and $\left\|\frac{g}{2^{n}}\right\|_{\Psi} \geq\left\|\frac{f^{(n)}}{4^{n}}\right\|_{\Psi}>$ $1, \forall n>0$, a contradiction with $g \in H^{\Phi} \subseteq L^{\Psi}$. 
(iii) $\Longrightarrow$ (i). We consider the martingale $f=\left(E\left(f \mid \mathcal{F}_{k}\right)\right)_{k \geq 0}$ as in the proof of Theorem 1, where $f=t \chi_{\left(0, \frac{1}{\left.2^{n}\right]}\right.}, t=\Psi^{-1}\left(2^{n}\right)$. From (iii) we have that $c_{2}\left\|f^{*}\right\|_{\Phi}>$ $\|f\|_{\Psi}=1$ and $E \Phi\left(c_{2} f^{*}\right)>1=E \Psi(f)$. Let $c_{4}=(2 \Phi(1) \vee 2) c_{2}$. By the convexity of $\Phi$ we have that $E \Phi\left(c_{4} f^{*}\right)>2 \Phi(1) \vee 2$. Notice that $\int_{0}^{1}\left|\left\{c_{4} f^{*}>s\right\}\right| \varphi(s) d s \leq \Phi(1)$, and then

$$
\int_{1}^{\infty}\left|\left\{c_{4} f^{*}>s\right\}\right| \varphi(s) d s>\frac{1}{2} \int_{0}^{\infty}\left|\left\{c_{4} f^{*}>s\right\}\right| \varphi(s) d s>1 .
$$

When $s \in\left(\frac{c_{4} t}{2^{n-k}}, \frac{c_{4} t}{2^{n-k-1}}\right](0 \leq k \leq n-1)$, we have that

$$
\frac{1}{s} \geq \frac{2^{n}}{c_{4} t} \cdot \frac{1}{2^{k+1}}=\frac{2^{n}}{c_{4} t} \cdot\left|\left\{c_{4} f^{*}>s\right\}\right|
$$

and when $s \in\left(0, \frac{c_{4} t}{2^{n}}\right]$, we have $\frac{1}{s} \geq \frac{2^{n}}{c_{4} t}$. Thus $\frac{1}{s} \geq \frac{2^{n}}{c_{4} t} \cdot\left|\left\{c_{4} f^{*}>s\right\}\right|, \forall s<c_{4} t$. From (10), the convexity of $\Psi$ and the fact $f^{*} \leq t$ we get

$$
\int_{1}^{c_{4} t} \frac{\varphi(s)}{s} d s \geq \frac{2^{n}}{c_{4} t} \int_{1}^{c_{4} t}\left|\left\{c_{4} f^{*}>s\right\}\right| \varphi(s) d s \geq \frac{2^{n}}{c_{4} t} \geq \frac{1}{2 c_{4}} \psi\left(\frac{t}{2}\right) .
$$

Thus for every $n \in N$, when $u \in\left(c_{4} \Psi^{-1}\left(2^{n}\right), c_{4} \Psi^{-1}\left(2^{n+1}\right)\right]$ we have

$$
\int_{1}^{u} \frac{\varphi(s)}{s} d s \geq \int_{1}^{c_{4} \Psi^{-1}\left(2^{n}\right)} \frac{\varphi(s)}{s} d s \geq \frac{1}{2 c_{4}} \psi\left(\frac{\Psi^{-1}\left(2^{n}\right)}{2}\right) \geq \frac{1}{4 c_{4}} \psi\left(\frac{u}{4 c_{4}}\right) ;
$$

hence

$$
\int_{1}^{t} \frac{\varphi(s)}{s} d s \geq c_{1} \psi\left(c_{1} t\right), \quad \forall t \geq c_{4} \Psi^{-1}(2)
$$

where $c_{1}=\frac{1}{4 c_{4}}$, which implies (i) and the proof is complete.

Remark. There is an example which shows that Theorem 3 is not true if we replace the nonnegative regular martingale in (ii) and (iii) by a regular martingale. To see this, let $\Phi(t)=t, \Psi(t)=t \log ^{+} t$, then $\Phi, \Psi$ satisfy (i). Consider the martingale $f=\left(E\left(4^{n} \chi_{\left(0, \frac{1}{2^{n+1}}\right]}-4^{n} \chi_{\left(\frac{1}{2^{n+1}}, \frac{1}{2^{n}}\right]} \mid \mathcal{F}_{k}\right)\right)_{k \geq 0}$ on $(0,1]$, where $\mathcal{F}_{k}=\sigma\left\{\left(0, \frac{1}{2^{k}}\right], 1 \leq\right.$ $k \leq n\}$. It is easy to check that $\|f\|_{\Psi}>\sqrt{n}\left\|f^{*}\right\|_{\Phi}$ when $n$ is big enough. The proof of (ii) $\Longrightarrow$ (iii) shows that $H^{\Phi} \nsubseteq L^{\Psi}$.

Corollary 3. Suppose that $\Phi, \Psi$ are nondecreasing convex functions defined on $[0, \infty)$ with $\Phi(0)=\Psi(0)=0$. Then the following statements are equivalent:

(i) There exist $s_{0}, c_{1}>0$ such that $\int_{1}^{t} \frac{\varphi(s)}{s} d s \geq c_{1} \psi\left(c_{1} t\right), \quad \forall t>s_{0}$.

(ii) There exists $c>0$ such that

$$
E \Psi(f) \leq c+c d_{\left(\mathcal{F}_{n}\right)}\left(\|f\|_{1} \vee c\right) E \Phi\left(c f^{*}\right)
$$

for every nonnegative regular martingale $f=\left(f_{n}\right)_{n \geq 0}$.

Proof. We only prove (i) $\Longrightarrow$ (ii). (ii) $\Longrightarrow$ (i) can be obtained from Theorem 3 . From the proof of Theorem 3, we have $E \Psi\left(c_{1} f\right) \leq c+d_{\left(\mathcal{F}_{n}\right)} E \Phi\left(f^{*}\right)$ for every nonnegative regular martingale $f$ with $\|f\|_{1} \leq 1$, where $c=\Psi\left(c_{1}\right)$. Now for a nonnegative martingale $f=\left(f_{n}\right)_{n \geq 0}$ adapted to $\left(\mathcal{F}_{n}\right)_{n \geq 0}$ with $\|f\|_{1}=2^{m}, m \in N$, consider the martingale $g=\left(g_{n}\right)_{n \geq 0}$ defined on $\left(\tilde{\Omega}, \tilde{\mathcal{F}}_{n}, \tilde{\mu}\right)=\left(\Omega \times(0,1], \mathcal{F}_{n} \times \sigma\left\{\left(0, \frac{1}{2^{k}}\right], 1 \leq k \leq\right.\right.$ $n\}, \mu \times \nu): g_{n}(\omega, t)=E\left(f \chi\left(0, \frac{1}{2^{m}}\right] \mid \tilde{\mathcal{F}}_{n}\right)$, where $\nu$ is Lebesque measure on $(0,1]$. Then $\|g\|_{1}=1$ and

$$
E \Psi\left(c_{1} g\right) \leq c+d_{\left(\tilde{\mathcal{F}}_{n}\right)} E \Phi\left(g^{*}\right)=c+2 d_{\left(\mathcal{F}_{n}\right)} E \Phi\left(g^{*}\right) .
$$


Notice that $E \Phi\left(g^{*}\right) \leq \frac{1}{2^{m}} E \Phi\left(f^{*}\right)+\sum_{1}^{m}\left(\frac{1}{2^{k}}\right) E \Phi\left(f_{k}^{*}\right) \leq E \Phi\left(f^{*}\right)$; we get

$$
\frac{1}{2^{m}} E \Psi\left(c_{1} f\right) \leq c+2 d_{\left(\mathcal{F}_{n}\right)} E \Phi\left(f^{*}\right) .
$$

For any $f$ with $\|f\|_{1}>1$, choose $c_{0}, m$ such that $2^{m-1}<\|f\|_{1} \leq 2^{m}, c_{0}=\frac{2^{m}}{\|f\|_{1}}<2$. From (11) we have

$$
\begin{aligned}
E \Psi\left(c_{1} f\right) & \leq E \Psi\left(c_{1} c_{0} f\right) \leq c\left\|c_{0} f\right\|_{1}+2 d_{\left(\mathcal{F}_{n}\right)}\left\|c_{0} f\right\|_{1} E \Phi\left(c_{0} f^{*}\right) \\
& \leq 2 c\|f\|_{1}+4 d_{\left(\mathcal{F}_{n}\right)}\|f\|_{1} E \Phi\left(2 f^{*}\right) .
\end{aligned}
$$

Thus for every nonnegative regular martingale $f$, we get

$$
E \Psi\left(c_{1} f\right) \leq 2 c\left(\|f\|_{1} \vee 1\right)+4 d_{\left(\mathcal{F}_{n}\right)}\left(\|f\|_{1} \vee 1\right) E \Phi\left(2 f^{*}\right)
$$

and

$$
E \Psi(f) \leq \frac{2 c}{c_{1}}\left(\|f\|_{1} \vee c_{1}\right)+\frac{4}{c_{1}} d_{\left(F_{n}\right)}\left(\|f\|_{1} \vee c_{1}\right) E \Phi\left(\frac{2 f^{*}}{c_{1}}\right) .
$$

Choose $c_{2}>c_{1}$ such that $\frac{4}{c_{1}} \Phi\left(\frac{2}{c_{1}} t\right)>\frac{2 c}{c_{1}}, \forall t \geq c_{2}$; we obtain

$$
E \Psi(f) \leq c_{3}+c_{4} d_{\left(\mathcal{F}_{n}\right)}\left(\|f\|_{1} \vee c_{2}\right) E \Phi\left(c_{5} f^{*}\right)
$$

where $c_{3}=\frac{2 c}{c_{1}} c_{2}, c_{4}=\frac{8}{c_{1}}, c_{5}=\frac{2}{c_{1}}$. Hence the assertion in (ii) is true.

\section{Some Equivalent CONDitions And EXAmples}

Corollary 1 shows that condition (8) is sufficient and necessary for the extension of Doob's inequality. In the following, we discuss the relationship between (8) and the condition $q_{\Phi}>1$ when $\Phi \in \Delta_{2}$ (i.e. there exist $c, t_{0}>0$ such that $\left.\Phi(2 t) \leq c \Phi(t), \forall t>t_{0}\right)$.

Lemma 3. If $\Phi$ is a nondecreasing convex function on $[0, \infty)$ with $\Phi(0)=0$, and there exists $c>0$ such that $\Phi(2 t) \leq c \Phi(t), \forall \Phi(t)>0$, then $q_{\Phi}>1$ if and only if there exists $c_{1}>0$ such that $\int_{0}^{t} \frac{\varphi(s)}{s} d s \leq c_{1} \varphi\left(c_{1} t\right), \forall t>0$.

Proof. First we prove the sufficiency. Notice that $\frac{\Phi(s)}{s^{2}} \leq \frac{1}{q_{\Phi}} \frac{\varphi(s)}{s}$; we get

$$
\int_{0}^{t} \frac{\varphi(s)}{s} d s \leq \frac{q_{\Phi}}{q_{\Phi}-1} \int_{0}^{t} \frac{\varphi(s)}{s}-\frac{\Phi(s)}{s^{2}} d s \leq \frac{q_{\Phi}}{q_{\Phi}-1} \frac{\Phi(t)}{t} \leq \frac{q_{\Phi}}{q_{\Phi}-1} \varphi(t) .
$$

Next we prove the necessity. Notice that the condition $\int_{0}^{t} \frac{\varphi(s)}{s} d s \leq c_{1} \varphi\left(c_{1} t\right)$ implies $\varphi(s) \downarrow 0($ as $s \rightarrow 0)$ and $\int_{0}^{t} \frac{\varphi(s)}{s} d s<\infty, \forall t>0$. Denote

$$
a_{k}=2^{k} \varphi\left(2^{k}\right)-\Phi\left(2^{k}\right)-\left[2^{k-1} \varphi\left(2^{k-1}\right)-\Phi\left(2^{k-1}\right)\right] \quad(-\infty<k<+\infty) ;
$$

we have

$$
2^{k-1}\left[\varphi\left(2^{k}\right)-\varphi\left(2^{k-1}\right)\right] \leq a_{k} \leq 2^{k}\left[\varphi\left(2^{k}\right)-\varphi\left(2^{k-1}\right)\right]
$$

and

$$
\sum_{k=-\infty}^{m} 2^{-k} a_{k} \leq \varphi\left(2^{m}\right)-\varphi(0) \leq \sum_{k=-\infty}^{m} 2^{-k+1} a_{k}
$$

Therefore,

$$
\Phi\left(2^{m}\right) \leq \sum_{k=-\infty}^{m-1} 2^{k} \varphi\left(2^{k+1}\right) \leq \sum_{k=-\infty}^{m-1} 2^{k} \sum_{i=-\infty}^{k+1} 2^{-i+1} a_{i} \leq \sum_{i=-\infty}^{m} 2^{m-i+1} a_{i} .
$$


On the other hand, we have

(16)

$$
\int_{0}^{2^{m}} \frac{\varphi(s)}{s} d s \geq \sum_{k=-\infty}^{m-1} 2^{k} \frac{\varphi\left(2^{k}\right)}{2^{k+1}} \geq \frac{1}{2} \sum_{k=-\infty}^{m-1} \sum_{i=-\infty}^{k} 2^{-i} a_{i}=\frac{1}{2} \sum_{i=-\infty}^{m-1} 2^{-i}(m-i) a_{i} .
$$

Notice that $\inf _{t>0} \frac{t \varphi(t)-\Phi(t)}{\Phi\left(\frac{t}{2}\right)}=0$ if $q_{\Phi}=1$. From (12) and (15) we get that $\forall j>1$, $\exists t_{j} \in\left(2^{n_{j}}, 2^{n_{j}+1}\right]$ such that $\Phi\left(\frac{t_{j}}{2}\right)>0$ and

$$
\left(\sum_{k=-\infty}^{n_{j}} 2^{n_{j}-k+1} a_{k}\right)^{-1} \sum_{k=-\infty}^{n_{j}} a_{k} \leq \frac{2^{n_{j}} \varphi\left(2^{n_{j}}\right)-\Phi\left(2^{n_{j}}\right)}{\Phi\left(2^{n_{j}}\right)} \leq \frac{t_{j} \varphi\left(t_{j}\right)-\Phi\left(t_{j}\right)}{\Phi\left(\frac{t_{j}}{2}\right)} \leq \frac{1}{2^{j}} .
$$

Then for $k_{0}=n_{j}-j+1$, we have

$$
\left(\sum_{k=k_{0}+1}^{n_{j}} 2^{n_{j}-k+1} a_{k}\right)^{-1} \sum_{k=-\infty}^{n_{j}} a_{k} \geq \frac{1}{2^{j-1}}, \quad\left(\sum_{k=-\infty}^{k_{0}} 2^{n_{j}-k+1} a_{k}\right)^{-1} \sum_{k=-\infty}^{n_{j}} a_{k} \leq \frac{1}{2^{j-1}} .
$$

Thus $\sum_{k=-\infty}^{n_{j}} 2^{-k} a_{k} \leq 2 \sum_{k=-\infty}^{k_{0}} 2^{-k} a_{k}$ and

$$
\frac{\varphi\left(\frac{t_{j}}{2}\right)}{\int_{0}^{2 t_{j}} \frac{\varphi(s)}{s} d s} \leq\left(\frac{1}{2} \sum_{k=-\infty}^{n_{j}}\left(n_{j}-k+1\right) 2^{-k} a_{k}\right)^{-1} \sum_{k=-\infty}^{n_{j}} 2^{-k+1} a_{k} \leq \frac{8}{\left(n_{j}-k_{0}+1\right)}=\frac{8}{j} .
$$

Therefore

$$
j \varphi\left(\frac{t_{j}}{2}\right) \leq 8 \int_{0}^{2 t_{j}} \frac{\varphi(s)}{s} d s \leq 8 c_{1} \varphi\left(2 c_{1} t_{j}\right)
$$

and

$$
j \Phi\left(\frac{t_{j}}{2}\right) \leq j \frac{t_{j}}{2} \varphi\left(\frac{t_{j}}{2}\right) \leq 4 c_{1} t_{j} \varphi\left(2 c_{1} t_{j}\right) \leq \Phi\left(6 c_{1} t_{j}\right), \forall j>0,
$$

a contradiction that proves the necessity.

Theorem 4. Let $\Phi$ be a nondecreasing convex function on $[0, \infty)$ with $\Phi(0)=0$ and $\Phi \in \Delta_{2}$. Then $\underline{\lim }_{t \rightarrow \infty} \frac{t \varphi(t)}{\Phi(t)}>1$ if and only if there exist some $s_{0}, c_{1}>0$ such that (8) holds.

Proof. Without loss of generality, we can assume that there exists $t_{0}>0$ such that $t \psi(t)>\Psi(t), \Psi(2 t) \leq c \Psi(t), \forall t>t_{0}$. Now, for any $s_{0}>t_{0}$, consider the function $\widetilde{\Psi}(t)=\Psi(t) \chi_{\left[s_{0}, \infty\right)}+\Psi\left(s_{0}\right)\left(\frac{t}{s_{0}}\right)^{\alpha} \chi_{\left[0, s_{0}\right)}$, where $\alpha=\frac{s_{0} \psi\left(s_{0}\right)}{\Psi\left(s_{0}\right)}>1$. Applying Lemma 3 to $\widetilde{\Psi}(t)$ we get

$$
\inf _{t>s_{0}} \frac{t \psi(t)}{\Psi(t)}>1 \text { if and only if } \exists c>0 \text { such that } \int_{s_{0}}^{t} \frac{\psi(s)}{s} d s<c \psi(c t), \forall t>s_{0} .
$$

The proof is finished.

The following are some examples which make the inequalities in this paper hold:

Example 1. For $1<p<\infty$, let

$$
\Phi(t)=\Psi(t)= \begin{cases}t, & t<1 \\ t^{p}, & t \geq 1\end{cases}
$$


In this case,

$$
\varphi(t)=\psi(t)= \begin{cases}1, & t<1 \\ p t^{p-1}, & t \geq 1\end{cases}
$$

Example 2. Let

$$
\Phi(t)=t, \Psi(t)=t \log ^{+} t .
$$

In this case

$$
\varphi(t)=1, \psi(t)=\left\{\begin{array}{cc}
0, & t<1 \\
1+\log ^{+} t, & t \geq 1 .
\end{array}\right.
$$

Example 3. Suppose that $n \geq 1$ and

$$
\Phi(t)=t\left(\log ^{n} t\right)^{+}, \quad \Psi(t)=t\left(\log ^{n+1} t\right)^{+} .
$$

In this case

$$
\begin{aligned}
& \varphi(t)=\left\{\begin{array}{cc}
0, & 0 \leq t<1, \\
n \log ^{n-1} t+\log ^{n} t, & t \geq 1, \\
0, & 0 \leq t<1,
\end{array}\right. \\
& \psi(t)=\left\{\begin{array}{cr}
(n+1) \log ^{n} t+\log ^{n+1} t, & t \geq 1 .
\end{array}\right.
\end{aligned}
$$

\section{REFERENCES}

[1] C. Dellacherie, Inegalites de convexite pour les processsus croissants et les sousmartingales, LNM, 721(1979), 371-377. MR 83f:60071

[2] D. Gilat, On the ratio of the expected maximum of a martingale and the $L^{p}$-norm of its last term, Israel J, Math., 63(1988), 270-280. MR 89m:60102

[3] C. Herz, $\mathrm{H}_{p}$-spaces of martingales, $0<p \leq 1$, Z.Wahrs. theorie, 28(1974), 189-205. MR 51:9189

[4] P. Imkeller, Two-parameter martingales and their quadratic variation. LNM, 1308(1988), Springer-Verlag. MR 89e:60098

[5] H. Kita, On Hardy-Littlewood maximal functions in Orlicz spaces, Math. Nachr, 183 (1997), 135-155. MR 98c:42016

[6] H. Kita, On maximal funcitions in Orlicz spaces, Proceedings of A.M.S. 124(1996), 3019-3025. MR 97b:42031

[7] H. Kita, On a converse inequality for maximal functions in Orlicz spaces, Studia Math., 118(1996), 1-10. MR 97g:42015

[8] R.L. Long, Martingale spaces and inequalities, Beijing:Peking Univ Press, 1993. MR 94j:60087

[9] T. Mei, P.D. Liu, Double $\Phi$-function inequality for nonnegative submartingales, Chinese Ann. of Math., 21(2000), 211-216. CMP 2000:14

[10] J. Neveu, Martingales a temps discret, Masson, Paris, 1972. MR 53:6728

[11] M.M. Rao, Z. D. Ren, Theory of Orlicz spaces, Marcel Dekker, New York, 1991. MR 92e: 46059

[12] E.M. Stein, Note on the class $L \log L$, Studia Math., 32(1969), 305-310. MR 40:799

College of Mathematics Sciences, Wuhan University, Hubei, 430072, People's RepubLIC OF CHINA

E-mail address: meitaosuizhou@263.net

Current address, November 2001-November 2002: Department of Mathematiques, U.F.R. des Sciences et Techniques, 16, Route de Gray, -F-25030 Besancon Cedex, France

College of Mathematics Sciences, Wuhan University, Hubei, 430072, People's RepubLIC OF CHINA

E-mail address: pdliu@whu.edu.cn 